\title{
A Flocking-Based Dynamical Systems Paradigm for Smart Power System Analysis
}

\author{
Jin Wei, Deepa Kundur, Takis Zourntos and Karen Butler-Purry \\ Department of Electrical \& Computer Engineering \\ Texas A\&M University, College Station, TX 77843, USA
}

\begin{abstract}
We propose a paradigm to model the cyber-physical interactions related to transient stability in a smart grid. In our multi-agent framework each node, representing both electrical and information system components, is modeled as having dynamics that synergistically describe physical and information couplings with neighboring agents. Physical behaviors are described through the application of swing equations to a reduced model of the electrical grid. Cyber-physical integrated action is formulated as a flocking control problem to achieve transient stability. Analysis and simulation demonstrate the potential of the paradigm to model cyber-physical smart grid dynamics as well as highlight strategies for effective distributed control.
\end{abstract}

\section{INTRODUCTION}

Electric power utilities currently reside in an environment of increasing power demand, market deregulation and a growing movement towards sustainability. Consequently, utilities have been driven to reduce operating margins pushing power systems to exhibit greater nonlinearity. Moreover the movement towards a smarter grid integrates sophisticated information systems within this already complex network. We assert that fundamental models that strengthen the scientific basis of smart power systems are needed to better understand the associated cyber-physical interactions.

In contrast to former papers that consider empirical models for cyber-physical interaction [1]-[4], we take on a more mathematical flavor. Moreover, we address the complex interplay amongst active elements such as generators and dynamic loads, in contrast to approaches that model individual generators and loads that connect via linearized lossless networks [5].

Recent work has proposed finding a balance between dynamical representations and models found in network science [6]-[10]. In this spirit, we build upon dynamical models of power systems recently employed in [8] and incorporate cyber intelligence and control behaviors by taking a flocking perspective commonly used to model large-scale natural phenomenon.

We assert that our multi-agent flocking-based approach has the following advantages. First, it enables the convenient integration of cyber (communications and control) systems within dynamical models of power system physics. Second, the structure of our models conveniently enables the study of the important transient stability problem. Third, the models of cyber system dynamics can be employed to gain insight on effective smart grid distributed communications and control strategies for system performance and stabilization. Moreover, our paradigm also can be employed to model the interplay of centralized power system control centers and supervisory control and data acquisition (SCADA) within emerging smart grid environments.

Our research carries the flavor of recent work by Dörfler and Bullo [8] and Feng et al. [11] by applying cooperative control strategies to power systems with the following distinctions. We incorporate cyber system dynamics into our formation thus enabling characterizations of cyber-physical dependencies of importance to smart grids. The flocking paradigm allows communications and control goals to be elegantly abstracted within models of the physical power system to aid in the study of system operation and control. Moreover we are able to relax the overdamped generator assumption of [8] through the effective design of cyber dynamics.

The next section introduces our multi-agent paradigm and transient stability. Section III reviews the principles of flocking as it relates to the transient stability. The proposed cyberphysical control protocol used to model dynamics is detailed in Section IV. Analysis and simulation are provided in Sections V and VI, respectively, followed by conclusions.

\section{Proposed Multi-Agent Paradigm}

\section{A. Cyber-Physical Integration}

Figure 1 presents our networked multi-agent-based framework that integrates both cyber and physical elements for systems such as the 3-Generator 9-bus WECC system considered later in this paper and even larger systems that we discuss in [12]. Our model is comprised of cyber-physical agents, each consisting of: (1) a dynamic node representing physical power system elements such as generators (or active loads that are not considered in this paper), (2) a phasor measurement unit (PMU) that acquires physical data from the dynamic node including phase angle and frequency of the associated active node, and (3) a local cyber-controller which obtains information from the PMU to compute a control signal that is applied to the generator node. The local controller and PMU are considered to be a cyber elements due to their data acquisition, communications and computation tasks.

Since our focus is on the transient stability problem, the objective of the local controller is to achieve generator frequency synchronization in the face of cyber-physical distur- 


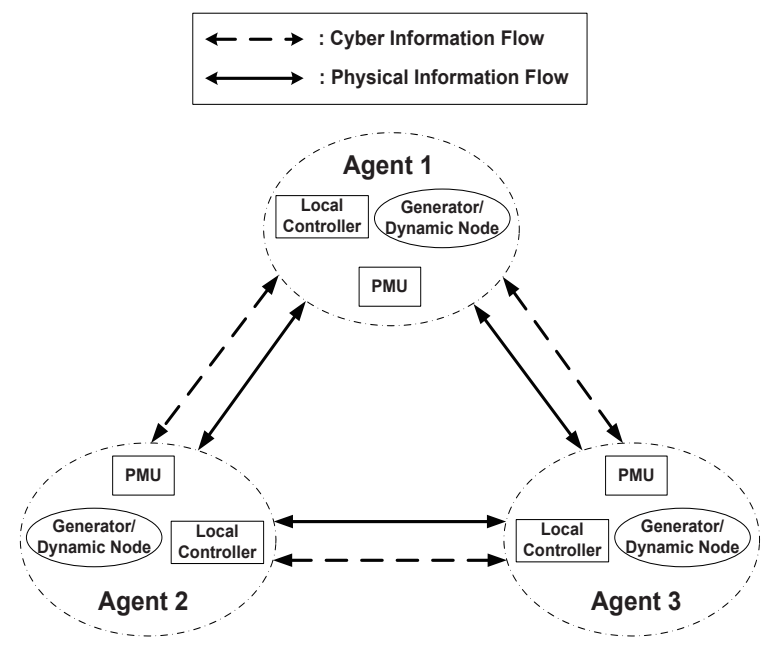

Fig. 1. Equivalent networked multi-agent dynamic system model.

bance. As such, the local controllers may require fast-acting external power sources in order to achieve their objectives. These sources in practice may include battery storage devices, renewable energy sources, plug-in hybrid electric vehicles (PHEVs) and flywheels, and may be separate for each agent. For convenience, PMU data is modeled as being sent to a data concentrator such that all agents can access the information to make effective control decisions. The reader should note that models in which PMUs directly communicate with one another would not change the paradigm. In fact, Fig. 1 presents the equivalent multi-agent model considering the effective information (cyber) and power (physical) flows.

\section{B. Dynamics and Interaction}

Assuming there are $N$ agents in general, each is assigned a coupled dynamical system equation to model the cyberphysical interactions. To describe the physical system, we make use of the well-known interconnected swing equations to describe rotor dynamics [13] of the Kron-reduced [14] power system as detailed by Dörfler and Bullo in [8] to give the following dynamical representation for each agent:

$M_{i} \dot{\omega}_{i}=-D_{i} \omega_{i}+P_{m, i}-\left|E_{i}\right|^{2} G_{i i}-\sum_{j=1}^{N} P_{i j} \sin \left(\theta_{i}-\theta_{j}+\varphi_{i j}\right)$

where $i \in\{1,2, \ldots, N\}$ represents the generator index, $\theta_{i}$ denotes the rotor phase angle measured with respect to a rotating frame reference at frequency $f_{0}=60 \mathrm{~Hz}, \omega_{i}=\dot{\theta}_{i}$ is the relative normalized frequency, $M_{i}>0$ and $D_{i}>0$ represent the generator inertia and the damping parameters, respectively, $E_{i}, P_{m, i}$ and $G_{i i}$ are the internal voltage, mechanical power input and equivalent shunt conductance of Generator $i$, respectively. $P_{i j}=\left|E_{i}\right|\left|E_{j}\right|\left|Y_{i j}\right|$ and $\varphi_{i j}=$ $\arctan \left(G_{i j} / B_{i j}\right)$ where $Y_{i j}, G_{i j}$ and $B_{i j}$ are the Kronreduced equivalent admittance, conductance and susceptance, respectively, between Generators $i$ and $j$.

The cyber network consisting of PMU information and local controllers is integrated into this framework through the injection of a control input to the right side of Eq. (1) to give:

$$
\begin{aligned}
M_{i} \dot{\omega}_{i}= & -D_{i} \omega_{i}+P_{m, i}-E_{i}^{2} G_{i i} \\
& -\sum_{j=1}^{N} P_{i j} \sin \left(\theta_{i}-\theta_{j}+\varphi_{i j}\right)+\underbrace{u_{i}}_{\text {control input }}
\end{aligned}
$$

where $u_{i}=P_{u, i}$ is the control signal for the $i$ th agent and is computed as a function of the data acquired by all the PMUs in the system, $\theta_{j}, \omega_{j}$ for $j \in\{1,2, \ldots, N\}$. The control can be interpreted as power injection for positive $P_{u, i}$ or absorption for negative $P_{u, i}$ at the corresponding generator buses from the fast-acting external power sources. Thus, it represents a cyberto-physical bridge whereby computation of $u_{i}$ is converted to active power flow.

Thus, the dynamics of Eq. (2) represents both cyber and physical interactions. Here the physical-to-cyber bridge exists at the measurement devices in which physical phase angle and frequency are converted to PMU data and the cyberto-physical bridge occurs when the cyber-computed control signal $u_{i}$ is applied (through power injection of $P_{u, i}$ ) into the generator network. The model of $u_{i}$ as a function of PMU data represents the contribution of the cyber network to the overall framework. For normal system operation $u_{i}=0$. However, when a disturbance strikes, $u_{i}$ will excite the system to reachieve (transient) stability. Computation of $u_{i}$ is dependent on the overall controller goals that we discuss next.

\section{Transient Stability}

We focus on controller design for transient stability (to model the cyber dynamics). It is well-known that transient stability describes the ability of a power system to remain in synchronism when subjected to large disturbances, such as transmission line faults and generator loss [13]. Achieving transient stability in a faulted system consists of maintaining both exponential frequency synchronization and phase angle cohesiveness through possible application of control strategies. Exponential frequency synchronization requires the frequencies of all the generators agree asymptotically with a common value $f_{0}$ typically set to $60 \mathrm{~Hz}$ and normalized in this formulation to 1 . Phase angle cohesiveness necessitates that the difference between the phase angle of each generator (band the center of inertia (COI) phase (defined below) should be below a pre-defined threshold typically chosen to be $5 \pi / 9$. Analytically, we can write these goals as:

1) Exponential frequency synchronization:

$$
\omega_{i}(t) \rightarrow 0, \text { as } t \rightarrow \infty
$$

where $\omega_{i}(t)$ is the normalized frequency of Generator $i$ at time $t$;

2) Phase angle cohesiveness:

$$
\left|\theta_{i}(t)-\theta_{C O I}(t)\right| \leq \gamma, \forall t
$$

where $\gamma$ is normally set to $5 \pi / 9$ in practice, and $\theta_{C O I}(t)$ is the COI phase angle defined as:

$$
\theta_{C O I}=\frac{\sum_{i=1}^{N} M_{i} \theta_{i}}{\sum_{i=1}^{N} M_{i}} .
$$


From Eqs. (4) and (5), it can be found that a sufficient condition for phase angle cohesiveness is that $\forall i, j \in\{1, \ldots, N\}$ :

$$
\left|\theta_{i}-\theta_{j}\right| \leq \frac{5 \pi}{9}
$$

\section{Flocking-BASed Cyber-Physical Modeling}

Let the state of each generator agent be given by $\left(\theta_{i}(t), \omega_{i}(t)\right)$. It is clear from the previous section that the physical coupling between agents is reflected by parameters $P_{i j}$ and $\varphi_{i j}$ of Eq. (2). The cyber coupling is reflected in the control signal $u_{i}$ that is a function of PMU data from neighboring generators. Given the self-regulation goals of the transient stability problem, we consider cyber-control between agents using deviations of their phase angle $\theta_{i}(t)-\theta_{j}(t)$ and frequency $\omega_{i}(t)-\omega_{j}(t)$ computed from the PMU measurements of local and neighboring generators. In doing this, we are able to recognize the analogies between the transient stability problem and that of flocking introduced in [15].

\section{A. Flocking Formation Control}

In a system comprised of a large number of coupled agents, flocking refers to an aggregate behavior amongst the entities to achieve a shared group objective. In [15], Reynolds introduced three heuristic rules that led to the creation of the first computer animation of flocking:

1) Flock Centering: agents attempt to stay close to nearby flockmates,

2) Collision Avoidance: agents avoid collisions with nearby flockmates,

3) Velocity Matching: agents attempt to match velocity with nearby flockmates.

Based on these three rules, Olfati-Saber [16] provided a framework for design and analysis of scalable distributed flocking algorithms using a double integrator model:

$$
\left\{\begin{array}{l}
\dot{\mathbf{q}}=\mathbf{p} \\
\dot{\mathbf{p}}=\mathbf{u},
\end{array}\right.
$$

where $\mathbf{q} \in \mathbb{R}^{N}$ is the position vector of the flockmates, $\mathbf{p} \in \mathbb{R}^{N}$ denotes the velocity vector, $\mathbf{u} \in \mathbb{R}^{N}$ represents the control signal, and $N$ is the size of the flock.

To achieve the objectives of flocking, the control signal $\mathbf{u}$ is comprised of three terms:

$$
\mathbf{u}=-\nabla V(\mathbf{q})-\mathbf{L} \cdot \mathbf{p}+F\left(\mathbf{p}, \mathbf{q}, \mathbf{p}_{r}, \mathbf{q}_{r}\right) .
$$

The first term is the gradient of a potential energy function $V(\mathbf{q})$ which characterizes system objectives and constraints. The second term represents a velocity consensus protocol where $\mathbf{L}$ is the Laplacian matrix associated with the flock communication graph. Finally, the third term models navigational feedback which is designed to ensure each agent tracks a reference $\left(\mathbf{p}_{r}, \mathbf{q}_{r}\right)$.

The stability of the control protocol described in Eq. (8) has been analyzed in [16] to provide the following sufficient conditions for stability: (1) $V(\mathbf{q})$ is a nonnegative continuously differentiable potential energy function that achieves the global minimum at a desired formation; (2) $\mathbf{L}$ is a standard
Laplacian matrix, which is positive semidefinite and has a zero rowsum [17]; (3) $F\left(\mathbf{p}, \mathbf{q}, \mathbf{p}_{r}, \mathbf{q}_{r}\right)$ is a linear combination of $\left(\mathbf{p}-\mathbf{p}_{r}\right)$ and $\left(\mathbf{q}-\mathbf{q}_{r}\right)$.

\section{B. Flocking and Transient Stability}

By studying the three flocking rules, we notice that flock centering and the velocity matching are analogous to the phase angle cohesiveness and the exponential frequency synchronization objectives in the transient stability problem, respectively. This analogy inspires us to reformulate the problem of transient stability as a task of flocking formation control.

\section{Proposed Cyber-Physical Control Protocol}

From Eq. (2) we delineate the cyber coupling (cyber $\S$ ) and physical coupling (phys $\S$ ) amongst agents as:

$$
\begin{aligned}
M_{i} \dot{\omega}_{i}= & -D_{i} \omega_{i}+P_{m, i}-E_{i}^{2} G_{i i} \\
& -\sum_{j=1}^{N} \underbrace{P_{i j}}_{\text {phys } \S} \sin (\theta_{i}-\theta_{j}+\underbrace{\varphi_{i j}}_{\text {phys }})+\underbrace{u_{i} .}_{\text {cyber } \S}
\end{aligned}
$$

Thus, the problem of representing the collective cyber-physical dynamics of the agents requires the effective design of $u_{i}$ to model the influence of the cyber network via distributed controllers common in emerging smart grid systems.

For development of the cyber control dynamics $u_{i}$, we make two assumptions. First, we assume that the rate of change of $u_{i}=P_{u, i}$ is much larger than that of the mechanical power input $P_{m, i}$ for each agent and the time span to recover transient stability is short; thus we treat $P_{m, i}$ as a constant. This assumption is reasonable for future smart grid systems where fast-response energy sources such as PHEV and flywheels will be available to inject and absorb energy for periods of brief control. Second, we assume that the problems of voltage regulation and transient stability are decoupled. This enables us to consider the voltage $E_{i}$ as a constant during controller excitation to re-achieve the transient stability.

Under these assumptions, computing the derivatives of the both sides of the equation above (or Eq. (2)), the system dynamics may be reformulated as:

$\left\{\dot{\theta}_{i}=\omega_{i}\right.$,

$\left\{\begin{array}{l}\dot{\theta}_{i}=\omega_{i}, \\ D_{i} \dot{\omega}_{i}=-M_{i} \ddot{\omega}_{i}-\sum_{j=1}^{N} P_{i j} \cos \left(\theta_{i}-\theta_{j}+\varphi_{i j}\right)\left(\omega_{i}-\omega_{j}\right)+\dot{u}_{i} .\end{array}\right.$

The system of Eq. (9) is not feedback linearizable due to the presence of the $M_{i} \ddot{\omega}_{i}$ term [18]. Moreover, it is not appropriate to apply singular perturbation analysis since in typical practice $M_{i} \in[2 \mathrm{~s}, 12 \mathrm{~s}] /\left(2 \pi f_{0}\right)$ and $D_{i} \in[1 \mathrm{~s}, 3 \mathrm{~s}] /\left(2 \pi f_{0}\right)$; thus, the perturbation parameter $\epsilon=M_{\max } / D_{\min } \in \mathcal{O}(10)$ [18]. In order to overcome this obstacle, we design $u_{i}$ as follows:

$u_{i}=-D_{i} \sum_{j \in \mathcal{N}_{i}} b_{i j}\left(\omega_{i}-\omega_{j}\right)-\sum_{j \in \mathcal{N}_{i}} g_{i j}\left(\theta_{i}-\theta_{j}\right)+h(\boldsymbol{\theta}, \boldsymbol{\omega})$

where $b_{i j}$ is a parameter used to facilitate singular perturbation as we discuss later, $g_{i j}>0$ is a control parameter, $\mathcal{N}_{i}$ denotes the index set of the neighbors of the $i$ th agent, and $h(\cdot): \mathbb{R}^{N} \times \mathbb{R}^{N} \rightarrow \mathbb{R}$ is a function of the vector $\boldsymbol{\theta}=\left\{\theta_{1}, \ldots, \theta_{N}\right\}$ and the vector $\boldsymbol{\omega}=\left\{\omega_{1}, \ldots, \omega_{N}\right\}$. 
Based on Eq. (10), we can rewrite the second line of Eq. (9) as follows:

$$
\begin{aligned}
& D_{i}\left(1+\sum_{j \in \mathcal{N}_{i}} b_{i j}\right) \dot{w}_{i}-D_{i} \sum_{j \in \mathcal{N}_{i}}\left(b_{i j} \dot{w}_{j}\right)= \\
& -M_{i} \ddot{\omega}_{i}-\sum_{j=1}^{N}\left[P_{i j} \cos \left(\theta_{i}-\theta_{j}+\varphi_{i j}\right)\right. \\
& \left.+g_{i j}\left(\theta_{i}-\theta_{j}\right)\right]\left(\omega_{i}-\omega_{j}\right)+\frac{d}{d t} h(\boldsymbol{\theta}, \boldsymbol{\omega})
\end{aligned}
$$

where we assign the parameters $\left\{b_{i j}\right\}$ to satisfy $\epsilon=M_{\max } /\left[D_{\min }\left(1+\sum_{j \in \mathcal{N}_{i}} b_{i j}\right)\right] \in \mathcal{O}(0.1)$. This enables us to utilize singular perturbation techniques to study the dynamics of our networked multi-agent system of Eq. (9) over a longer time scale. We can rewrite Eq. (9) in the vector form as follows:

$$
\left\{\begin{array}{l}
\dot{\boldsymbol{\theta}}=\boldsymbol{\omega}, \\
\dot{\boldsymbol{\omega}}=-\mathbf{M}^{-1}(\mathbf{L}+\mathbf{G}) \boldsymbol{\omega}+\mathbf{M}^{-1} \boldsymbol{\nu} .
\end{array}\right.
$$

where $\mathbf{M}=\mathbf{D} \Psi, \mathbf{\Psi}=(\mathbf{I}+\mathbf{B}), \mathbf{I}$ is an identity matrix, $\mathbf{D}=\operatorname{diag}\left[D_{1}, \ldots, D_{N}\right], \mathbf{B}=\left[b_{i j}\right]$ and $\mathbf{G}=\left[g_{i j}\right]$ are our proposed $N \times N$ cyber control matrices, $\mathbf{L}$ is a $N \times N$ physical coupling matrix whose elements can be represented as:

$$
l_{i j}= \begin{cases}\sum_{j \neq i} P_{i j} \cos \left(\theta_{i}-\theta_{j}+\varphi_{i j}\right), & \text { if } i=j ; \\ -P_{i j} \cos \left(\theta_{i}-\theta_{j}+\varphi_{i j}\right), & \text { if } i \neq j,\end{cases}
$$

and $\nu$ is a $N$-dimensional column vector such that the $i$ th element is given by $\nu_{i}=\frac{d}{d t} h\left(\theta_{i}, \omega_{i}\right)$. It is straightforward to show that the matrices $\mathbf{L}, \mathbf{G}$ and $\mathbf{B}$ all have the trivial eigenvalue 0 with corresponding eigenvector $\mathbf{1}$, and the matrix $\Psi$ is a row stochastic nonnegative matrix which has a trivial eigenvalue 1 and the corresponding eigenvector 1 [17].

Since the nonlinear dynamical system of Eq. (11) is feedback linearizable, we can define a new control vector $\widetilde{\mathbf{u}}$ and rewrite the equivalent reduced order model as:

$$
\left\{\begin{array}{l}
\dot{\theta}=\omega \\
\dot{\omega}=\widetilde{\mathbf{u}}
\end{array}\right.
$$

Furthermore, we can represent the relationship between the original control vector $\mathbf{u}$ and the new control vector $\widetilde{\mathbf{u}}$ as:

$$
\dot{\mathbf{u}}=\widetilde{\mathbf{D}}(\widetilde{\mathbf{u}}+\mathbf{L} \boldsymbol{\omega}),
$$

where $\widetilde{\mathbf{D}}$ is a $N \times N$ diagonal matrix with $\widetilde{d}_{i i}=1 / D_{i}$.

\section{A. Design by Analogy to Flocking}

Eq. (13) represents a double integrator system analogous to Eq. (7) known to model the standard dynamics of flockings. By setting $\widetilde{\mathbf{u}}$ to the following form we thus ensure flocking formation and hence transient stability of the power network:

$$
\widetilde{\mathbf{u}}=\underbrace{-\mathbf{M}^{-1} \nabla V(\boldsymbol{\theta})}_{\text {phase cohesiveness }}-\underbrace{\widetilde{\mathbf{L}} \boldsymbol{\omega}+\mathbf{M}^{-1} F\left(\boldsymbol{\omega}, \boldsymbol{\omega}_{r}\right)}_{\text {frequency synchronization }},
$$

where $V(\boldsymbol{\theta})$ represents the potential energy function to guarantee that the phase angle differences between pairs of agents are bounded, $\nabla V(\boldsymbol{\theta})$ is its associated gradient with respect to $\boldsymbol{\theta}, \widetilde{\mathbf{L}}$ is the effective Laplacian matrix that ensures frequency consensus (i.e., agents' frequencies converge to a common value), and $F(\cdot)$ is the navigation feedback designed to "lead" the frequencies to converge to the desired value $\boldsymbol{\omega}_{r}$; typically relative frequency is normalized such that $\boldsymbol{\omega}_{r}=\mathbf{0}$.

1) Potential Energy Function: Based on the sufficient condition of Eq. (6), we consider the following potential energy for our control scheme:

$$
V(\theta)=\frac{1}{2} \sum_{i} \sum_{j \neq i} \psi\left(\theta_{i}-\theta_{j}\right),
$$

where $\psi(\cdot)$ is a pairwise attractive potential defined as:

$$
\psi(z)= \begin{cases}0, & \text { if }|z| \leq \frac{5 \pi}{9} \\ c_{1}\left(z^{2}-\frac{25 \pi^{2}}{81}\right)^{2}, & \text { otherwise }\end{cases}
$$

where $c_{1}$ is a parameter to control the penalty level induced.

2) Effective Laplacian: Based on Eqs. (11), (13), and (14), we submit that the effective Laplacian matrix $\widetilde{\mathbf{L}}$ can be assigned as follows (and incorporates both cyber and physical interactions):

$$
\widetilde{\mathbf{L}}=\mathbf{M}^{-1}(\mathbf{L}+\mathbf{G}) .
$$

Since $\mathbf{L}$ and $\mathbf{G}$ both have the trivial eigenvalue 0 with associated eigenvector $\mathbf{1}$ (i.e., $\mathbf{L} \cdot \mathbf{1}=\mathbf{0}$ and $\mathbf{G} \cdot \mathbf{1}=\mathbf{0}$ ), we deduce: $\widetilde{\mathbf{L}} \cdot \mathbf{1}=\mathbf{M}^{-1}(\mathbf{L}+\mathbf{G}) \cdot \mathbf{1}=\mathbf{M}^{-1}(\mathbf{L} \cdot \mathbf{1}+\mathbf{G} \cdot \mathbf{1})=\mathbf{0}$. Thus the elements of $\widetilde{\mathbf{L}}$ have a rowsum of zero expressed also as:

$$
\widetilde{l}_{i i}=-\sum_{j=1, j \neq i}^{N} \widetilde{l}_{i j}
$$

From Gershgorin's Theorem [19], we reason that the eigenvalues of $\widetilde{\mathbf{L}}$ are located in the union of the $N$ discs:

$$
\bigcup_{i=1}^{N}\left\{z \in C:\left|z-\widetilde{l}_{i i}\right| \leq \sum_{j=1, j \neq i}^{N}\left|\widetilde{l}_{i j}\right|\right\} .
$$

Using Eqs. (18) and (19) we obtain that:

$$
\bigcup_{i=1}^{N}\left\{z \in C:\left|z+\sum_{j=1, j \neq i}^{N} \widetilde{l}_{i j}\right| \leq \sum_{j=1, j \neq i}^{N}\left|\widetilde{l}_{i j}\right|\right\} .
$$

In order to guarantee the frequency convergence of the multiple agents, the Laplacian matrix $\widetilde{\mathbf{L}}$ must be positive semidefinite (PSD), i.e. all its nonzero eigenvalues are located in the right half plane. From Eq. (20), we deduce that a sufficient condition to ensure $\widetilde{\mathbf{L}}$ is PSD is $\widetilde{l}_{i j}<0$ where $i \neq j$. Furthermore, to simplify the controller design, we assume that the cyber communication graph is undirected which (coupled with the fact that the Kron-reduced physical graph is undirected) implies that the integrated cyber-physical graph is undirected thus constraining $\widetilde{\mathbf{L}}$ to be symmetric. 
3) Cyber Control Matrix B: The main function of the cyber matrix $\mathbf{B}$ is to ensure that the effective singular perturbation parameter $\epsilon=M_{\max } /\left[D_{\min }\left(1-\sum_{j=1, j \neq i}^{N} b_{i j}\right)\right] \in \mathcal{O}(0.1)$, where $M_{\max }$ is the maximum value of $\left\{M_{i}\right\}, D_{\min }$ is the minimum value of $\left\{D_{i}\right\}, b_{i j}$ is the $i j$ th element of $\mathbf{B}$, and typically $M_{\max } / D_{\min } \in \mathcal{O}(10)$ for practical generators. Also, since $\boldsymbol{\Psi}=\mathbf{I}+\mathbf{B}$ has the trivial eigenvalue 1 with corresponding eigenvector $\mathbf{1}$, the design of the cyber control matrix $\mathbf{B}$ has the additional constraint:

$$
b_{i i}=-\sum_{j=1, j \neq i}^{N} b_{i j} \in \mathcal{O}(100) .
$$

Letting $\mathbf{S}=\boldsymbol{\Psi}^{-1}$ and $\widehat{\mathbf{L}}=\mathbf{L}+\mathbf{G}$, we can rewrite Eq. (17) as:

$$
\widetilde{\mathbf{L}}=\mathbf{S} \widetilde{\mathbf{D}} \widehat{\mathbf{L}} \text {. }
$$

Thus, we have that:

$$
\widetilde{l}_{i j}=\sum_{k=1}^{N} \frac{s_{i k} \widehat{l}_{k j}}{D_{k}}
$$

where $\widetilde{l}_{i j}, s_{i k}$, and $\widehat{l}_{k j}$ are the elements of the matrices $\widetilde{\mathbf{L}}, \mathbf{S}$, and $\widehat{\mathbf{L}}$, respectively.

It can be shown that if a matrix is row stochastic and has only non-positive off-diagonal elements, then its inverse matrix is a row stochastic nonnegative matrix; we do not include the proof in this paper. Based on this, we have that $\mathbf{S}=\boldsymbol{\Psi}^{-1}$ is a row stochastic nonnegative matrix. Therefore $0 \leq s_{i k} \leq 1$ for all $i, k \in 1,2, \ldots, N$ and $\mathcal{D}=1 /\left(\sum_{k=1}^{N} D_{k}^{-1}\right)$ is a positive constant. Thus, $\mathcal{D} \tilde{l}_{i j}$ is a weighted average of the elements on the $j$ th column of $\hat{\mathbf{L}}$. We can deduce that as long as there is at least one negative element in each column of $\widehat{\mathbf{L}}$, we can design $\mathbf{G}$ to ensure $\widetilde{l}_{i j}<0$ for all $i \neq j$, which guarantees that $\widetilde{\mathbf{L}}$ is PSD as analyzed above.

Therefore, based on the properties of the matrix B developed above, we assign the elements of $\mathbf{B}$ as follows:

$$
b_{i j}= \begin{cases}100, & \text { if } i=j ; \\ -\frac{100}{N-1}, & \text { otherwise. }\end{cases}
$$

4) Cyber Control Matrix G: Based on the definition of $\mathbf{L}$ in Eq. (12), we observe that after a physical fault in the power system (which can be instigated by either a cyber or physical vulnerability), instability may ensue such that the phase angle different cannot asymptotically converge to achieve phase angle cohesiveness and $\mathbf{L}$ will have nonnegative off-diagonal elements. The main objective of the cyber control matrix $\mathbf{G}$ is to actively bring back phase cohesiveness in the presence of such physical disruption by ensuring that $\widehat{\mathbf{L}}$ is always symmetric and has at least one negative element in each column.

For symmetric Laplacians the convergence rate of consensus is determined by the second smallest eigenvalue. In order to guarantee the convergence rate of the multi-agents' frequencies and reduce the computational complexity, we design all the off-diagonal elements of the matrix $\widehat{\mathbf{L}}$ to be bounded by a negative upper bound $\rho$. To tradeoff cost of the power transmission $P_{u}$ and the convergence rate of the frequencies, we choose $\rho \in[-1,-0.5]$.

5) Optimization of $P_{u, i}:$ In order to minimize transmission power $P_{u, i}$ (to meet transmission capacity limitations or reduce cost) while ensuring convergence rate for consensus (for performance), we consider design of an optimal G. As previously discussed we assume that cyber information flow is undirected which implies that the effective Laplacian matrix $\widetilde{\mathbf{L}}$ is symmetric. Based on Eq. (17), we observe $\mathbf{M} \widetilde{\mathbf{L}}=\mathbf{L}+\mathbf{G}$. Since B is designed to have identical off-diagonal elements and $\widetilde{\mathbf{L}}$ is symmetric, it follows that $\mathbf{M} \widetilde{\mathbf{L}}$ and subsequently $\widehat{\mathbf{L}}=\mathbf{L}+\mathbf{G}$ are symmetric.

We dynamically determine the cyber control matrix $\mathbf{G}$ to minimize the power transmission $P_{u, i}$ by developing an optimization model. Since at each time step, in the control protocol, the gradient-based, navigational feedback and cyber control matrix $\mathbf{B}$ terms are all predetermined, minimizing the total power transmission $P_{u, i}$ is equivalent to minimizing the power transmission $p_{g, i}$ introduced solely by matrix $\mathbf{G}$ where

$$
p_{g, i}=\sum_{j=1}^{N} g_{i j}\left(\theta_{i}-\theta_{j}\right) .
$$

In order to balance and minimize the power transmission required for each agent, we design the objective function for the optimization model as follows:

$$
p_{g}=\sum_{i=1}^{N}\left\|\sum_{j=1}^{N} g_{i j}\left(\theta_{i}-\theta_{j}\right)\right\|_{1},
$$

where $\|\cdot\|_{1}$ denotes the 1-norm. Furthermore, we set the constraints to ensure that all the off-diagonal elements of $\widehat{\mathbf{L}}$ are bounded by the upper bound $\rho$.

We illustrate our optimization model on the 3-Generator 9Bus WECC system. For this power system, $\mathbf{L}$ and $\mathbf{G}$ are both $3 \times 3$ matrices. All the elements $l_{i j}$ of $\mathbf{L}$ can be calculated. Considering that $\widehat{\mathbf{L}}$ is symmetric and $\mathbf{G}$ has a trivial eigenvalue 0 with corresponding eigenvector $\mathbf{1}$, we obtain that there are only three independent parameters for $\mathbf{G}$, which are the $(1,2)$ th, $(1,3)$ th and $(2,3)$ th matrix elements. Let the 3dimensional vector $\mathbf{X}=\left[x_{1}, x_{2}, x_{3}\right]^{T}$ consist of these three independent elements. Then we can represent the $i j$ th element of the matrix $\mathbf{G}$ as:

$$
\left\{\begin{array}{l}
g_{11}=-x_{1}-x_{2}, \\
g_{12}=x_{1}, \\
g_{13}=x_{2}, \\
g_{21}=l_{12}-l_{21}+x_{1}, \\
g_{22}=l_{21}-l_{12}-x_{1}-x_{3}, \\
g_{23}=x_{3} \\
g_{31}=l_{13}-l_{31}+x_{2}, \\
g_{32}=l_{23}-l_{32}+x_{3}, \\
g_{33}=l_{31}-l_{13}+l_{32}-l_{23}-x_{2}-x_{3} .
\end{array}\right.
$$

If we define $\mathbf{A}_{1}$ as:

$$
\mathbf{A}_{1}=\left(\begin{array}{ccc}
\theta_{1}-\theta_{2} & \theta_{1}-\theta_{3} & 0 \\
\theta_{2}-\theta_{1} & 0 & \theta_{2}-\theta_{3} \\
0 & \theta_{3}-\theta_{2} & \theta_{3}-\theta_{1}
\end{array}\right),
$$


we can formulate the objective function as follows:

$$
\begin{aligned}
p_{g} & =\|\mathbf{A}(1,:) \mathbf{X}\|_{1}+\left\|\mathbf{A}(2,:) \mathbf{X}+\left(l_{12}-l_{21}\right)\left(\theta_{1}-\theta_{2}\right)\right\|_{1} \\
& +\left\|\mathbf{A}(3,:) \mathbf{X}+\left(l_{13}-l_{31}\right)\left(\theta_{3}-\theta_{2}\right)+\left(l_{23}-l_{33}\right)\left(\theta_{3}-\theta_{1}\right)\right\|_{1}
\end{aligned}
$$

Defining another vector $\mathbf{A}_{2}=\left[\rho-l_{12}, \rho-l_{13}, \rho-l_{23}\right]$, we can formulate the optimization model as:

$$
\begin{aligned}
& \min _{\mathbf{X} \in \mathbb{R}^{3}} \quad p_{g} \\
& \text { s.t. } \quad \mathbf{X} \prec \mathbf{A}_{2},
\end{aligned}
$$

where $p_{g}$ is given by Eq. (22).

Based on the above analysis, we have the following result:

$\dot{\mathbf{u}}=-\sum_{j=1, j \neq i}^{N} \phi\left(\theta_{i}-\theta_{j}\right) \mathbf{1}-\mathbf{G} \boldsymbol{\omega}-\mathbf{B} \dot{\boldsymbol{\omega}} \cdot \mathbf{D}-c_{2}\left(\boldsymbol{\omega}-\boldsymbol{\omega}_{r}\right)$,

where $c_{2}$ is the navigational feedback parameter chosen as 1 in our work, $\boldsymbol{\omega}_{r}=\mathbf{0}$ denotes the normalized reference frequency and $\mathbf{D}=\left[D_{1}, D_{2}, D_{3}\right]^{T}$. By integrating the both sides of Eq. (24), we can formulate $\mathbf{u}$, which represents the power transmission $P_{u}$ between the fast-reacting power source and the synchronized generators, as:

$\mathbf{u}=-\sum_{j=1, j \neq i}^{N}\left[\int_{t_{0}}^{t} \phi\left(\theta_{i}-\theta_{j}\right) d t\right] \mathbf{1}-\mathbf{G} \boldsymbol{\theta}-\mathbf{B} \boldsymbol{\omega} \cdot \mathbf{D}-c_{2}\left(\boldsymbol{\theta}-\boldsymbol{\theta}_{0}\right)$,

where $t_{0}$ is the time to activate our control protocol and $\boldsymbol{\theta}_{0}$ is the constant term in $\int_{t_{0}}^{t} \boldsymbol{\omega} d t$.

6) Linear Navigation: To reduce control complexity, we assign the following linear navigation feedback term: $F\left(\boldsymbol{\omega}, \boldsymbol{\omega}_{r}\right)=c_{2} \boldsymbol{\omega}$, where $c_{2}=1$.

\section{CYBER DYNAMICS AND STABILITY}

Since our cyber-physical modeling framework aims to address the transient stability problem in smart grid systems, we analyze the controller dynamics in order to deduce its ability to provide regulation in the presence of disruption. We emphasize that the control $u_{i}$ enables a framework to model large-scale distributed cyber-control for transient stability. Moreover, it can provide essential insights into the design of distributed control in future power systems beyond the scope of this paper.

Letting $\widehat{\mathbf{u}}=\mathbf{M} \widetilde{\mathbf{u}}$, we rewrite the dynamics described in Eq. (13) as follows:

$$
\left\{\begin{array}{l}
\dot{\theta}=\omega \\
\mathbf{M} \dot{\omega}=\widehat{\mathbf{u}} .
\end{array}\right.
$$

where $\widehat{\mathbf{u}}=-\nabla V-(\mathbf{L}+\mathbf{G}) \boldsymbol{\omega}-c_{2} \boldsymbol{\omega}$. In order to analyze the stability of our proposed framework, we define the following Lyapunov function $H$ :

$$
H=\frac{1}{2} \boldsymbol{\omega}^{T} \mathbf{M} \boldsymbol{\omega}+\mathbf{V}
$$

It is clear that that $H(\mathbf{0}, \mathbf{0})=0$ and $H(\boldsymbol{\theta}, \boldsymbol{\omega})>0$ for $\forall(\boldsymbol{\theta}, \boldsymbol{\omega}) \neq(\mathbf{0}, \mathbf{0})$. By calculating the derivative of $H$ along the dynamics derived in Eq. (11), we obtain:

$$
\begin{aligned}
\dot{H} & =\boldsymbol{\omega}^{T} \mathbf{M} \dot{\boldsymbol{\omega}}+\boldsymbol{\omega}^{T} \nabla V, \\
& =\boldsymbol{\omega}^{T} \widehat{\mathbf{u}}+\boldsymbol{\omega}^{T}\left(-\widehat{\mathbf{u}}-\mathbf{L} \boldsymbol{\omega}-\mathbf{G} \boldsymbol{\omega}-c_{2} \boldsymbol{\omega}\right), \\
& =-\boldsymbol{\omega}^{T}\left(\mathbf{L}+\mathbf{G}+c_{2} \mathbf{I}\right) \boldsymbol{\omega} .
\end{aligned}
$$

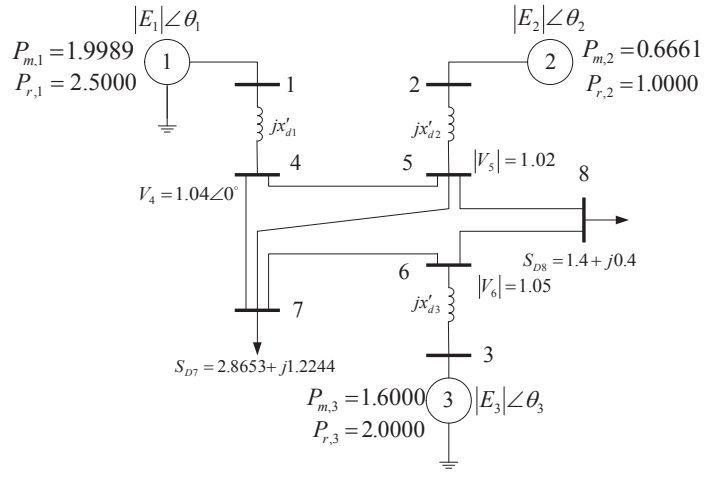

Fig. 2. WECC 9-bus power system

Based on our proposed framework, $\widehat{\mathbf{L}}=\mathbf{L}+\mathbf{G}$ is the effective Laplacian matrix which is PSD and $c_{2}>0$. Therefore, $\mathbf{L}+\mathbf{G}+c_{2} \mathbf{I}$ is a Positive Definite (PD) matrix. Using the property of PD matrices, we deduce $\boldsymbol{\omega}^{T}\left(\mathbf{L}+\mathbf{G}+c_{2} \mathbf{I}\right) \boldsymbol{\omega}>0$. Thus, $\dot{H}<0$ in Eq. (26) demonstrating Lyapunov stability of the proposed framework.

\section{Simulation}

We demonstrate the performance of our flocking-based controller structure in achieving transient stability for two case studies on the 3-generator, 9-bus WECC system of Fig. 2. MATLAB/Simulink is employed for simulations. In each case we assume that a physical fault occurs at time $t=0 \mathrm{~s}$ and the associated line is removed at $t=0.3 \mathrm{~s}$. Then, the control is activated at $t=0.4 \mathrm{~s}$. The distributed controllers determine $u_{i}=P_{u, i}$ as detailed in the previous section.

In order to demonstrate the utility of the proposed framework for wide area monitoring systems, the normalized impedance of the transmission line is increased from $0.1 j$ (standard for the WECC system) to $0.35 j$. The increase in impedance reduces the physical couplings amongst generators hence making the transient stability problem more challenging.

\section{A. Simulation Case Study I}

The system disturbance consists of a 3-phase short circuit at Bus 6 of Fig. 2. Fig. 3 shows the rotor frequencies, phase angles and phase angle differences over a period of $8 \mathrm{~s}$ when no control is applied. Instability is clearly evident in all plots (e.g., phase angle differences diverge greater than $5 \pi / 9$ ).

Fig. 4 (note: scales differ significantly from Fig. 3) demonstrates performance when the proposed flocking-based control is activated at $t=0.4 \mathrm{~s}$. The power transmission limit for the fast-acting grid is set to $\mu=P_{u, i} / P_{r, i} \leq \frac{4}{5}$ where $P_{r, i}$ is the rated power. The power transmission $P_{u}=\left(P_{u, 1}, P_{u, 2}, P_{u, 3}\right)$ is shown in Fig. 5. We observe that a capacity limit on the transmission line is simulated through the constraint $\mu \leq \frac{4}{5}$. Even though clipping of the control signal occurs transient stability is still achieved.

\section{B. Simulation Case Study II}

In the second study, a 3-phase short circuit occurs on the middle of Line $4-5$ of Fig. 2. Fig. 6 provides the resulting 

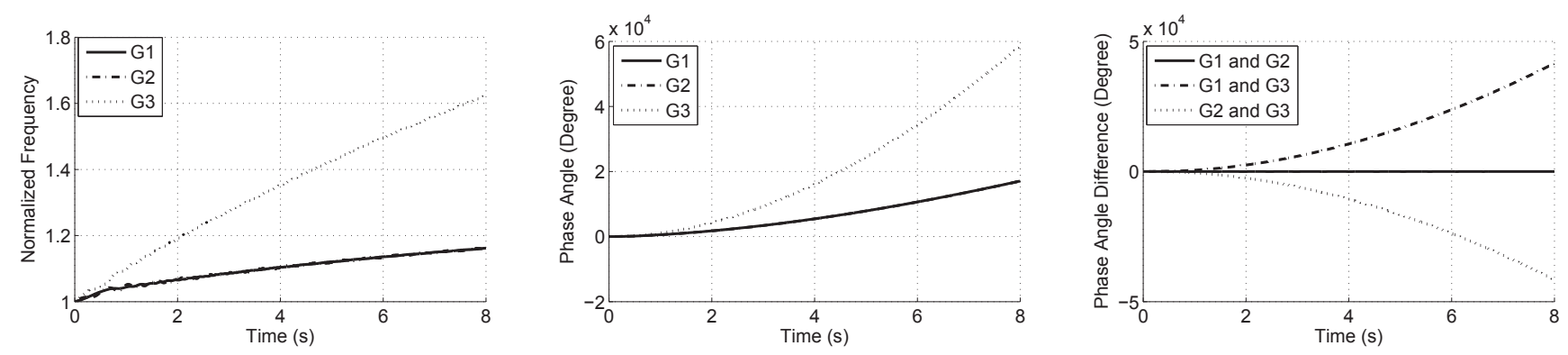

Fig. 3. Normalized rotor frequencies, phase angles, phase angle differences versus time without our proposed protocol.
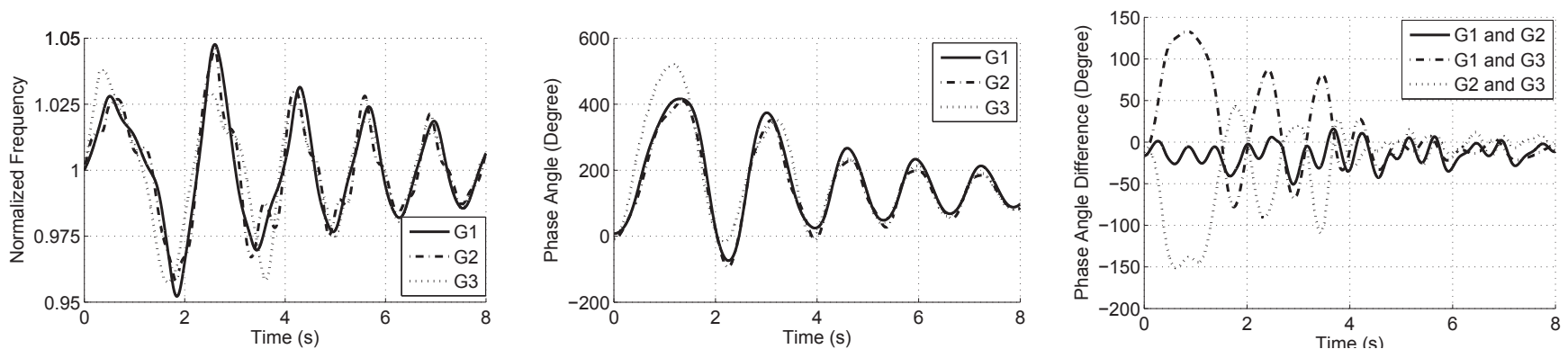

Fig. 4. Normalized frequencies, phase angles, phase angle differences versus time by using our proposed protocol.

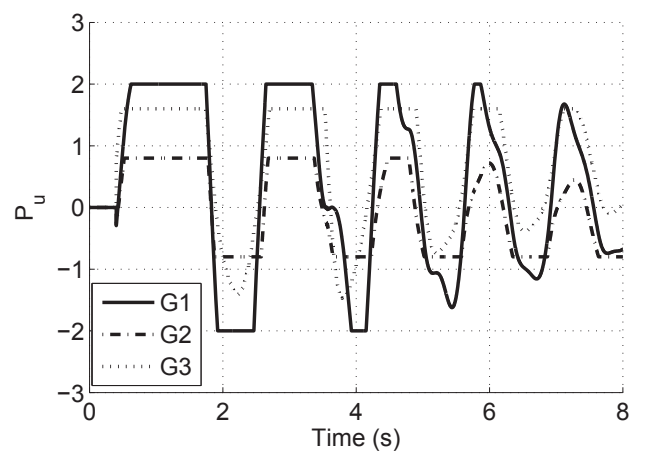

Fig. 5. Power transmission $P_{u}$ between the generators and the fast-reacting power grid in the presence of our proposed protocol.

rotor frequencies, phase angles and phase differences which demonstrate clear instability.

When the control is applied at $t=0.4 \mathrm{~s}$ and the power transmission parameter is set to $\mu \leq \frac{4}{5}$, stability in the face of this severe fault and control clipping is achieved as shown in Figs. 7 and 8 where $P_{u}$ is under the capacity constraint that $\mu \leq \frac{4}{5}$.

The reader should note that the MATLAB results presented in this paper are consistent with a separately synthesized model of the cyber-physical system in SimPowerSystems.

\section{CONCLUSIONS}

We have investigated a multi-agent dynamical system framework for modeling cyber-physical smart grid interactions related to the transient stability problem. Each agent consists

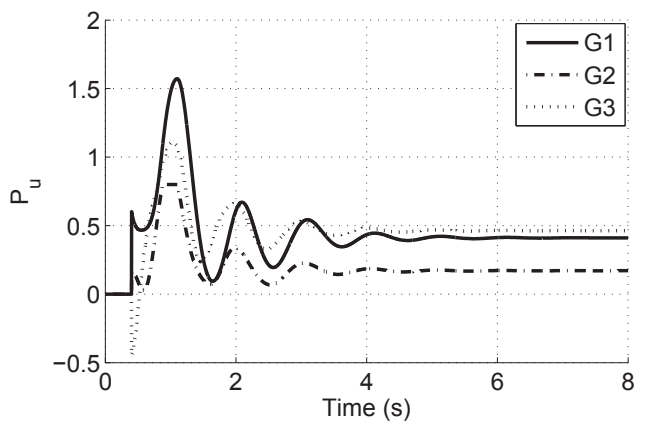

Fig. 8. Power transmission $P_{u}$ between the generators and the fast-reacting power grid in the presence of our proposed protocol.

of a synchronous power generator, PMU and local controller. Access to a fast-acting power grid enables application of power control for transient stability. The physical system operation is described through the application of swing equations to a kronreduced power network. The cyber system operation, which includes communications and control tasks, is integrated with the physical through a flocking control paradigm. We have demonstrated the elegant way in which cyber and physical couplings are described in the dynamical system equations of each agent. Moreover, simulations show the potential of the approach for modeling and providing insights on distributed smart grid strategies for transient stability. Ongoing work, focuses on modeling the fast-reacting grid using flywheel dynamics to determine appropriate constrains on the power control signal $P_{u}$. Future work will consider modeling chal- 

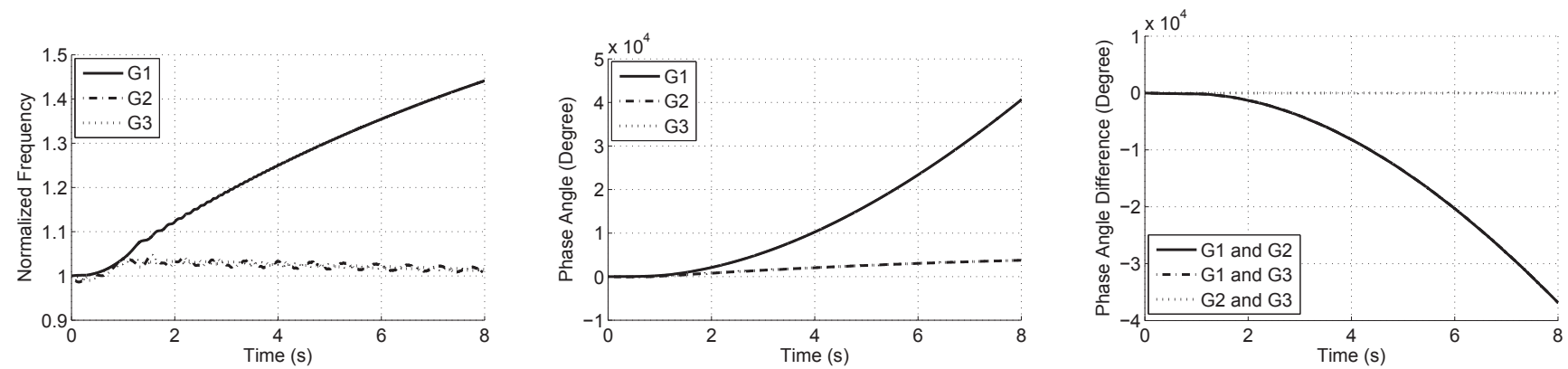

Fig. 6. Normalized rotor frequencies, phase angles, phase angle differences versus time without our proposed protocol.
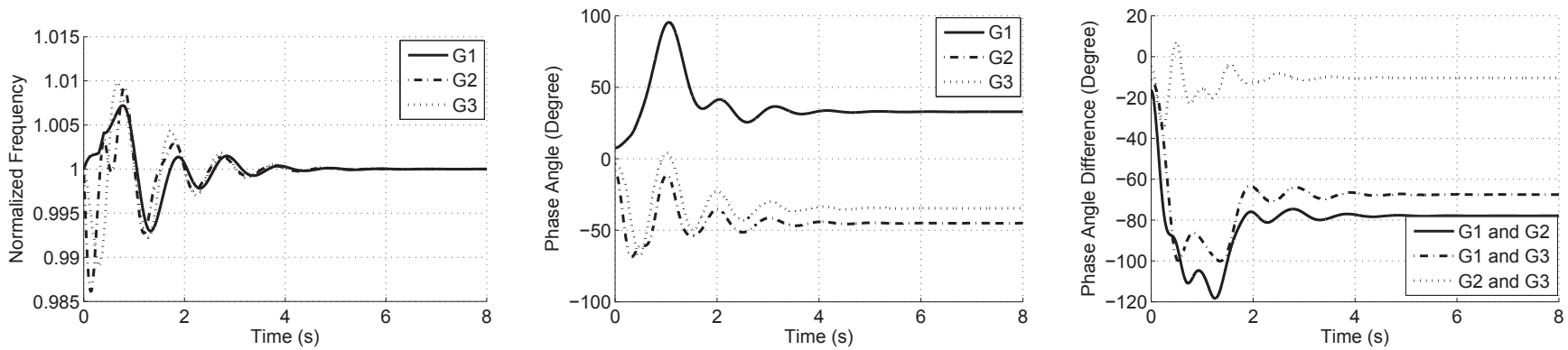

Fig. 7. Normalized frequencies, phase angles, phase angle differences versus time by using our proposed protocol.

lenges for dynamic loads in combination with PMU and local control as agents and strategies to group system components into agents as to minimize computational and control energy overhead.

\section{ACKNOWLEDGEMENTS}

Research funding was provided by the U.S. National Science Foundation under grant ECCS-1028246 and the Norman Hackerman Advanced Research Program Project Number 000512-0111-2009. The authors thank Xianyong Feng and Salman Mashayehk for stimulating discussions and comments during development of the work and Xianyong Feng for a preliminary version of the MATLAB simulation code.

\section{REFERENCES}

[1] D. D. Dudenhoeffer, M. R. Permann, S. Woolsey, R. Timpany, C. Miller, A. McDermott, and M. Manic, "Interdependency modeling and emergency response," in Proc. 2007 Summer Computer Simulation Conference, July 2007, pp. 1230-1237.

[2] B. Rozel, M. Viziteu, R. Caire, N. Hadjsaid, and J.-P. Rognon, "Towards a common model for studying critical infrastructure interdependencies," in Proc. IEEE Power and Energy Society General Meeting - Conversion and Delivery of Electrical Energy in the 21st Century, Pittsburgh, Pennsylvania, July 2008, pp. 1-6.

[3] N. HadjSaid, C. Tranchita, B. Rozel, M. Viziteu, and R. Caire, "Modeling cyber and physical interdependencies - application in ICT and power grids," in Proc. IEEE Power Systems Conference and Exposition, March 2009, pp. 1-6.

[4] J. Stamp, A. McIntyre, and B. Ricardson, "Reliability impacts from cyber attack on electric power systems," in Proc. IEEE Power Systems Conference and Exposition, March 2009, pp. 1-8.

[5] M. Ilic, L. Xie, U. Khan, and J. Moura, "Modeling future cyber-physical energy systems," in Proc. IEEE Power Engineering Society General Meeting, Pittsburg, PA, July 2008, pp. 1-9.
[6] D. J. Hill and G. Chen, "Power systems as dynamic networks," in Proc. IEEE International Symposium on Circuits and Systems (ISCAS), May 2006, pp. 722-725.

[7] V. Fioriti, S. Ruzzante, E. Castorini, E. Marchei, and V. Rosato, "Stability of a distributed generator network using the kuramoto models," in Critical Information Infrastructure Security, 2009, pp. 14-23.

[8] F. Dörfler and F. Bullo, "Synchronization and transient stability in power networks and non-uniform kuramoto oscillators," in Proc. American Control Conference, June-July 2010, pp. 930-937.

[9] D. Kundur, X. Feng, S. Liu, T. Zourntos, and K. Butler-Purry, "Towards a framework for cyber attack impact analysis of the electric smart grid," in Proc. IEEE International Conference on Smart Grid Communications (SmartGridComm), Gaithersburg, Maryland, October 2010, pp. 244 249.

[10] Y. Susuki, I. Mezić, and T. Hikihara, "Coherent swing instability of power grids," Nonlinear Science, February 2011.

[11] X. Feng, K. Butler-Purry, T. Zourntos, and H.-M. Chou, "Multiagent system-based real-time load management for NG IPS ships in high/medium voltage level," in Proc. IEEE Power Systems Conference and Exposition, March 2011, pp. 1-8.

[12] J. Wei and D. Kundur, "Two-tier hierarchical cyber-physical security analysis framework for smart grid," in Proc. IEEE Power Engineering Society General Meeting, invited, to appear, 2012.

[13] P. Kundur, Power System Stability and Control. McGraw-Hill, 1994.

[14] A. R. Bergen and V. Vittal, Power Systems Analysis. Prentice Hall, 1999.

[15] C. Reynolds, "Flocks, herds, and schools: a distributed behavioral model," Computer Graphics, vol. 21, no. 4, pp. 25-34, July 1987.

[16] R. Olfati-Saber, "Flocking for multi-agent dynamic systems: Algorithms and theory," IEEE Transactions on Automatic Control, vol. 51, no. 3, pp. 401-420, March 2006.

[17] C. D. Meyer, Matrix Analysis and Applied Linear Algebra. SIAM, 2001.

[18] H. Khalil, Nonlinear Systems. Prentice-Hall, 2002.

[19] R. A. Horn and C. R. Johnson, Topics in Matrix Analysis. Cambridge University Press, 1991. 